\title{
INFLUÊNCIA DA PRIVAÇÃO VISUAL NO TESTE DE UMA REPETIÇÃO MÁXIMA E NA PREDIÇÃo dE CARGA
} INFLUENCE OF VISUAL DEPRIVATION IN ONE REPETITION MAXIMUM TEST AND LOAD PREDICTION

\author{
Saulo Costa1, Dihogo Gama de Matos $^{2}$, Aldo Coelho Silva1, Felipe Aidar', Bernardo Rodrigues Minelli³, \\ Ingi Klain², Mauro Lucio Mazini Filho' \\ 1 Pós Graduação em Fisiologia do Exercício e Treinamento Desportivo - Centro Universitário de Volta Redonda - RJ - Brasil \\ 2 Universidade de Trás os Montes e Alto Douro - Vila Real - Portugal \\ 3 Programa de Pós Graduação Stricto Sensu em Enfermagem e Biociências - PpgEnfBio - Universidade Federal do Estado do Rio de Janeiro
}

Data de entrada do artigo: 04/07/2012

Data de aceite do artigo: 02/04/2013

\section{RESUMO}

Objetivo: O objetivo deste estudo foi comparar se a privação visual, quando incluída no teste de 1-RM, influencia de forma significativa a carga deslocada, o número de repetições e a percepção subjetiva de esforço. Método: Participaram 11 voluntários do gênero masculino, idade 23,64 $\pm 2,42$ anos; peso corporal 73,76 \pm 9,37 kg; estatura $174 \pm 0,06 \mathrm{~cm}$ e IMC $24,35 \pm 2,63 \mathrm{~kg} / \mathrm{m}^{2}$ ). Foram realizados os exercícios Leg Press $45^{\circ}$ (LP) e Supino Reto (SR). Os dados foram coletados em dois momentos, no primeiro momento foram realizados os testes de 1-RM sem privação visual (SPV) e com privação visual (CPV), e posteriormente adotou-se um percentual de carga de $85 \%$ de 1-RM (SPV e CPV). Resultados: Força Absoluta no SR e LP (SPV) =80,18 + 6,72 kg e 312 + 28,48 kg; (CPV)= $87,19 \pm 7,50$ e $355,82 \pm 29,26$. Força Relativa no SR e LP (SPV)= 1,10 $\pm 0,10$ e 4,28 $\pm 0,62 ;(C P V)=1,19 \pm 0,11$ e $4,89 \pm 0,68$. Número de repetições a $85 \%$ de $1 R M$ no SR e LP $(S P V)=4,82 \pm 0,87$ e $14,36 \pm 0,81 ;(C P V)=5,92 \pm$ 0,98 e 15,18 \pm 0,60. Percepção Subjetiva de esforço a 85\% de 1-RM no SR e LP (SPV)= 8,82 $\pm 0,40$ e 8,91 $\pm 0,30$; $(\mathrm{CPV})=8,36 \pm 0,50$ e 8,45 \pm 0,52. Conclusão: os resultados indicam que a privação visual, quando incluída no teste de 1-RM, permite um aumento no deslocamento da carga absoluta e da carga relativa, proporcionando um maior numero de repetições e uma menor percepção subjetiva de esforço.

Palavras-chave: Privação Visual, força muscular e treinamento de força

\section{ABSTRACT}

Objective: The aim of this study was to compare if visual deprivation, when included in the 1-RM test, significantly influences the load shifted, the number of repetitions and perceived exertion. Method: Participated 11 male volunteers, age 23,64 $\pm 2,42$ years, body weight 73,76 $\pm 9,37 \mathrm{~kg}$, height $174 \pm 0,06 \mathrm{~cm}$ and BMl 24,35 $\left.\pm 2,63 \mathrm{~kg} / \mathrm{m}^{2}\right)$. The exercises accomplished were Leg Press (LP) and Flat Bench (FB). Data were collected on two occasions, the first time the tests were performed 1-RM without visual deprivation (WVD) and visual deprivation (VD), and later took up a load percentage of 85\% 1-RM (WVD and VD). Results: Absolute Strength in FB and LP (WVD) $=80.18 \pm$ $6.72 \mathrm{~kg}$ and $312+28.48 \mathrm{~kg} ;(\mathrm{VD})=87,19 \pm 7,50 \mathrm{~kg}+355,82 \pm 29,26 \mathrm{~kg}$. Relative Strength in FB and LP (WVD) = $1,10 \pm 0,10$ and $4,28 \pm 0.62 ;(V D)=1,19 \pm 0,11$ and $4,89 \pm 0,68$. Number of repetitions at $85 \% 1$ RM in FB and LP $(W V D)=4,82 \pm 0,87$ and $14,36+0,81(V D)=5,92+0,98$ and $15,18+0,60$. Perceived exertion at 85\% 1-RM in FB and $L P(W V D)=8,82 \pm 0,40$ and 8,91 $\pm 0,30 ;(V D)=8,36+0,50$ and $8.45 \pm 0,52$. Conclusion: the results indicate that visual deprivation, when included in the 1-RM allows an increase in the displacement of the load absolute and relative load, providing a greater number of repetitions and a lower perceived exertion.

Keywords: Privation Visual, muscle strenght, strenght training 


\section{INTRODUÇÃO}

Exercício com pesos é uma atividade física sistematizada em que se deve atentar as variáveis como volume, intensidade, frequência, duração, recuperação, ordem dos exercícios, equipamentos e tipos de treinamento 1 . A forma pela qual essas variáveis são manipuladas resulta em efeitos diferenciados no aprimoramento da força $e$ suas variáveis (potência muscular, hipertrofia, força pura, resistência de força). Em posicionamento institucional direcionado aos adultos saudáveis, o $\mathrm{ACSM}^{2}$ apresentou uma extensa revisão sobre os modelos de progressão do treinamento, envolvendo as variáveis da prescrição.

O Treinamento de Força (TF) assume papel importante nos sistemas neuromuscular, cardiovascular e metabólico $^{3,4}$. Haskell et $\mathrm{al}^{5}$., recomendam o treinamento de força, como parte integrante de um programa de aptidão física para qualquer idade. Para a população idosa é um importante meio para uma boa qualidade de vida, podendo melhorar a capacidade funcional, diminuir o de risco de quedas, melhorar o equilíbrio e auxiliar a caminhada ${ }^{6,7}$.

Alguns autores ${ }^{8,9}$ afirmam que o total de carga utilizado é a variável mais importante no treinamento de força. Reforçando esta ideia, Fleck et $\mathrm{al}^{10}$., acreditam que a intensidade e a carga utilizada em um exercício específico são alguns dos mais importantes componentes do treinamento de exercícios com pesos. A intensidade do treinamento, geralmente, está ligada ao percentual de carga máxima, que ocorre através do teste de uma repetição máxima (1-RM) ${ }^{11}$. Alguns estudos propõem que para obter ganhos de hipertrofia e força não se deve utilizar menos de $60 \%$ de 1-RM, sendo que na maioria dos trabalhos, $70-85 \%$ para o trabalho de hipertrofia, e 90-100\% para ganhos de força pura ${ }^{12}$.

O teste de 1-RM é usado como medida diagnóstica de deslocamento de carga ou como parâmetro para a prescrição e monitoramento de um exercício. Ainda cabe mencionar, que o teste de 1-RM, é considerado "padrão ouro" pela literatura ${ }^{13}$. Há uma diversidade de variáveis que podem intervir no teste de 1-RM, superestimando ou subestimando o resultado da carga obtida, como: a influência do estimulo verbal, efeito da desidratação, programas de flexibilidade, a técnica utilizada durante o teste, a familiarização e a privação visual ${ }^{14,15}$. Com relação ao uso da privação da visão, poucos trabalhos se apresentam na literatura.

Sendo a privação visual uma variável interveniente que pode aumentar a carga deslocada durante um teste de força máxima, deve-se então buscar respostas para sua interferência. Até o presente, a literatura apresenta algumas lacunas relacionadas a privação visual e o treinamento de força, como a sua relação com a percepção subjetiva de esforço e o número de repetições realizadas. Com isso, objetivo deste estudo foi comparar se a privação visual, quando incluída no teste de 1-RM e na predição de carga, influencia de forma significativa a carga deslocada (força absoluta e relativa), o número de repetições e a percepção subjetiva de esforço (PSE).

\section{MATERIAIS E MÉTODOS}

A amostra foi composta por 11 voluntários do gênero masculino, idade 23,64 $\pm 2,42$ anos; peso corporal 73,76 \pm 9,37 kg; estatura $174 \pm 0,06 \mathrm{~cm}$ e índice de massa Corporal (IMC) $24,35 \pm 2,63 \mathrm{~kg} / \mathrm{m}^{2}$ ), selecionados aleatoriamente. Quanto às medidas antropométricas foi realizada a medida de massa corporal, através de balança (Filizola, Brasil) para aferir o peso em quilogramas (KG), com precisão de $0,1 \mathrm{Kg}$. Também foi aferida a estatura, através de estadiômetro Sanny (Sanny, Brasil), com precisão de $0,1 \mathrm{~cm}$. As variáveis antropométricas foram coletadas seguindo o sugerido pela $\mathrm{WHO}^{16}$.

Para melhor objetivar os resultados da amostra foram utilizados alguns critérios de exclusão tais como: portadores de lesões osteomioarticulares nos últimos seis meses e realização de quaisquer tipo de cirurgias articulares nos últimos doze meses.

Para inclusão no estudo, os participantes deveriam ser fisicamente ativos (com atividade física regular, pelo menos três vezes por semana) e praticantes de exercícios resistidos há pelo menos 12 meses. Os procedimentos adotados seguiram as normas de ética em pesquisas com humano, de acordo com às Normas Sobre Pesquisa Envolvendo Seres Humanos do Comitê de Ética da Universidade Castelo Branco com o número de protocolo 0127/2008, que constam na Resolução n 196/96 do Conselho Nacional de Saúde, de 10 de outubro de 1996, que são as diretrizes e normas regulamentadoras de pesquisas envolvendo seres humanos. Os voluntários assinaram o Termo de Consentimento Livre e Esclarecido (TCLE), depois de informadas sobre todos os procedimentos a serem realizados durante o estudo.

Os voluntários não tiveram acesso ao total de deslocamento de carga antes dos términos de todos os testes. O conhecimento da carga poderia causar uma diminuição da motivação intrínseca nos procedimentos do estudo, fazendo que $\mathrm{o}$ indivíduo perdesse o rendimento ${ }^{17}$.

Os dados foram coletados em dois momentos, no primeiro momento foram realizados os testes de 1-RM (sem e com privação visual), e posteriormente adotou-se um percentual de carga de $85 \%$ de 1-RM (sem e com privação visual). As etapas foram divididas em seis dias não consecutivos, com intervalos de 72 horas entre uma mensuração e outra. A coleta de dados constou das seguintes etapas: dia 1: Medida de massa corporal e estatura, e logo após foi aplicado o teste 1-RM, sem a privação visual com o objetivo de determinar a carga máxima nos aparelhos supino reto (SR) e leg press $45^{\circ}$ (LP); dia 2: Aplicação do teste de 1-RM para confiabilidade de carga (re-teste); dia 3: Teste de 1-RM com privação visual (uso de venda nos olhos) nos 
exercícios SR, LP; dia 4: Teste de confiabilidade de carga (re-teste com uso de vendas); dia 5: Com a obtenção das cargas no teste de 1-RM foi adotado uma carga no percentual de $85 \%$ de 1-RM e aplicado nos exercícios (SR e LP), sendo neste primeiro momento sem o uso de venda, o voluntário realizou o maior número possível de repetições até a falha concêntrica. A predição de carga de $85 \%$ de 1-RM foi baseada no teste de 1-RM sem a privação visual tanto para o exercício (SR e LP) com e sem venda. Encerrada a fase acima, após 2 minutos the era indagado sobre a percepção subjetiva de esforço (PSE) através da tabela de OMNI RES, com descritores visuais, verbais e índices numéricos de 0 a 10. Cabe ressaltar que todos os participantes do estudo durante duas semanas anteriormente aos testes com objetivo de familiarização com a tabela, utilizaram-na dentro de seus programas de treino ${ }^{18}$. Quanto à execução do movimento, só houve controle da fase excêntrica, sendo este tempo de 4 segundos estabelecidos por um metrônomo da marca Seiko, modelo DM50 que sinalizava a 60 (bpm). A dinâmica do movimento aconteceu da seguinte forma: Ao final da fase excêntrica o voluntário recebia um sinal para que realizasse a fase concêntrica o mais rápido possível; dia 6: O procedimento foi idêntico ao $5^{\circ}$ dia, entretanto, utilizou-se o uso de vendas nos olhos.

Nos testes de 1-RM, tanto com ou sem privação visual, a força relativa foi calculada pela razão da carga máxima deslocada $\div$ peso corporal $(\mathrm{kg})$.

O protocolo adotado para a realização do teste de 1-RM foi o preconizado por Chagas et al ${ }^{11}$. Foram permitidas até seis tentativas para o individuo encontrar sua carga máxima em uma única repetição. O tempo de intervalo entre uma tentativa e outra foi de 5 minutos. A carga máxima foi atingida em no máximo quatro tentativas. A carga adotada foi a última que o indivíduo conseguiu realizar uma repetição, sem êxito na segunda.

Durante o teste de 1-RM e de predição de carga ( $85 \%$ de 1-RM) nos exercícios SR e LP, o tempo de recuperação entre um exercício e outro oscilou entre 15-20 minutos e com intervalos de 72 horas para a realização do próximo teste. Foi usado o próprio aparelho para o aquecimento, com 20 repetições com o peso escolhido pelo avaliado ${ }^{12}$.
Visando redimir e minimizar a margem de erro nos testes de 1-RM e sobre o percentual de $85 \%$ de 1-RM foram adotadas algumas medidas padronizadas:

a) Instruções padronizadas foram estabelecidas antes dos testes, de modo que o avaliado soubesse de toda a rotina que estava envolvida nas coletas de dados.

b) o avaliado foi instruído sobre a técnica da execução dos exercícios.

c) o avaliador estava atento quanto à posição adotada pelo praticante no momento da medida, pois pequenas variações no posicionamento das articulações poderiam acionar outros músculos, levando a escores errôneos.

d) os participantes receberam estímulos verbais a fim de manter o alto nível de rendimento nos testes;

\section{ANÁLISE ESTATÍSTICA}

Para a análise dos dados foi utilizado o pacote estatístico SPSS versão 17.0. O coeficiente de relação intra e intertestes nos testes de 1-RM, para a reprodutibilidade da carga, foi verificado pela Correlação de Pearson. Para efeito comparativo entre o testes de 1-RM e da predição de carga ( $85 \%$ de 1-RM) sem ou com a privação visual, foi utilizado o teste $T$ student, com índice de significância $p<0,05$. Os valores foram expressos em média, desvio padrão, escore máximo, escore mínimo.

\section{RESULTADOS}

Cabe ressaltar que, sobre a reprodutibilidade da medida da carga no teste e re-teste de 1-RM, foram encontrados valores relevantes de correlação intrateste 0,97 e 0,95 para SR e LP, respectivamente. Os coeficientes de relação intertestes sem privação visual (SPV) e com privação visual (CPV) foram de 0,97 para SR e de 0,99 para LP.

É apresentado na tabela 1 a relação da carga deslocada (força absoluta) no teste de 1-RM SPV e CPV nos

Tabela 1: Relação da força absoluta $(\mathrm{kg})$ no teste de 1-RM SPV e CPV nos exercícios SR e LP.

\begin{tabular}{|c|c|c|c|c|}
\hline Variáveis (kg) & Média(kg) & Escore Máximo(Kg) & Escore Mínimo(kg) & Teste $T$ student \\
\hline \multicolumn{4}{|c|}{ Supino Reto (SR) } & \multirow{3}{*}{${ }^{*} 0,03$} \\
\hline SPV & $80,18 \pm 6,72$ & 90 & 72 & \\
\hline CPV & $87,19 \pm 7,50$ & 98 & 78 & \\
\hline \multicolumn{4}{|c|}{ Leg Press $45^{\circ}(\mathrm{LP})$} & \multirow{3}{*}{${ }^{*} 0,001$} \\
\hline SPV & $312 \pm 28,48$ & 382 & 284 & \\
\hline $\mathrm{CPV}$ & $355,82 \pm 29,26$ & 426 & 324 & \\
\hline
\end{tabular}

*diferença significativa $\mathrm{p}$ valor $<0,05$. 
exercícios SR e LP. Percebe-se que no exercício SR quando a carga foi aferida sem o uso de vendas - que o deslocamento foi de $80,18 \pm 6,72 \mathrm{~kg}$, e que com a privação visual, ocorreu um aumento para $87,19 \pm 7,50 \mathrm{~kg}$, perfazendo um percentual de $8,74 \%$. No teste $T$ student observa-se uma diferença significativa entre os testes de ${ }^{*} 0,03$. No exercício LP, teste CPV, também é demonstrado aumento de volume de carga deslocado comparado com o SPV. Sendo o último com valores de $312 \pm 28,48 \mathrm{~kg}$ para a média de $355,82 \pm 29,26 \mathrm{~kg}$, consolidando assim um acréscimo de $14,04 \%$. No teste $T$ student, $p={ }^{*} 0,001$.

É apresentado na tabela 2 a relação da força relativa no testes SPV e CPV nos exercícios SR e LP. Houve melhoras significativas nos dois exercícios no teste CPV. No teste $T$ student, o valor foi de ${ }^{*} 0,04$ para ambos os exercícios.
Na tabela 3, apresenta-se a relação do número de repetições sobre uma carga de $85 \%$ de 1 -RM nos testes SPV e CPV, nos exercícios SR e LP. Observa-se um aumento de $22,82 \%$ no número repetições no exercício SR comparando os testes sem venda $(4,82 \pm 0,87)$ e com venda $(5,92 \pm 0,98)$. Também é possível observar que houve uma diferença significativa entre os testes de $p={ }^{*} 0,02$. Seguindo nesta mesma direção, nota-se o mesmo comportamento no que diz respeito ao exercício LP: aumento de $5,71 \%$ no número de repetições relacionando os testes SPV $(14,36 \pm 0,81)$ e CPV $(15,18 \pm 0,60)$. Pelo teste $T$ student foi verificada um valor de ${ }^{*} 0,01$.

A tabela 4 demonstra relação da PSE (OMNI-RES) em função da carga de $85 \%$ de 1-RM. Conforme observamos na tabela, ocorreu uma melhora da PSE

Tabela 2: Relação da força relativa (carga deslocada $\div$ peso corporal) no teste de 1-RM SPV e CPV nos exercícios SR e LP.

\begin{tabular}{|c|c|c|c|c|}
\hline Variáveis (kg) & Média(kg/Kg) & Escore Máximo & Escore Mínimo & Teste $T$ student \\
\hline \multicolumn{4}{|c|}{ Supino Reto (SR) } & \multirow{3}{*}{${ }^{*} 0,04$} \\
\hline SPV & $1,10 \pm 0,10$ & 1,22 & 0,91 & \\
\hline CPV & $1,19 \pm 0,11$ & 1,32 & 0,99 & \\
\hline \multicolumn{4}{|c|}{ Leg Press $45^{\circ}(\mathrm{LP})$} & \multirow{3}{*}{${ }^{*} 0,04$} \\
\hline SPV & $4,28 \pm 0,62$ & 5,40 & 3,87 & \\
\hline $\mathrm{CPV}$ & $4,89 \pm 0,68$ & 6,16 & 4,46 & \\
\hline
\end{tabular}

*diferença significativa $p$ valor $<0,05$.

Tabela 3: Relação do número de repetições em função de uma carga de 85\%1RM SPV e CPV nos exercícios SR e LP.

\begin{tabular}{|c|c|c|c|c|}
\hline Variáveis (kg) & $\begin{array}{l}\text { Média } \\
\text { (repetições) }\end{array}$ & $\begin{array}{l}\text { Escore Máximo } \\
\text { (repetições) }\end{array}$ & $\begin{array}{l}\text { Escore Mínimo } \\
\text { (repetições) }\end{array}$ & Teste $T$ student \\
\hline \multicolumn{5}{|c|}{ Supino Reto (SR) } \\
\hline SPV & $4,82 \pm 0,87$ & 7 & 4 & \multirow[t]{2}{*}{${ }^{*} 0,02$} \\
\hline CPV & $5,92 \pm 0,98$ & 7 & 4 & \\
\hline \multicolumn{4}{|c|}{ Leg Press $45^{\circ}(\mathrm{LP})$} & \multirow{3}{*}{${ }^{*} 0,01$} \\
\hline SPV & $14,36 \pm 0,81$ & 15 & 13 & \\
\hline CPV & $15,18 \pm 0,60$ & 16 & 14 & \\
\hline
\end{tabular}

*diferença significativa $p$ valor $<0,05$.

Tabela 4: Relação da PSE (OMNI RES) em função de uma carga de 85\%1RM SPV e CPV nos exercícios SR e LP.

\begin{tabular}{|c|c|c|c|c|}
\hline Variáveis (kg) & Média & Escore Máximo & Escore Mínimo & Teste $T$ student \\
\hline \multicolumn{4}{|c|}{ Supino Reto (SR) } & \multirow{3}{*}{${ }^{*} 0,03$} \\
\hline SPV & $8,82 \pm 0,40$ & 9 & 8 & \\
\hline CPV & $8,36 \pm 0,50$ & 9 & 8 & \\
\hline \multicolumn{4}{|c|}{ Leg Press $45^{\circ}(\mathrm{LP})$} & \multirow{3}{*}{${ }^{*} 0,02$} \\
\hline SPV & $8,91 \pm 0,30$ & 9 & 8 & \\
\hline CPV & $8,45 \pm 0,52$ & 9 & 8 & \\
\hline
\end{tabular}

*diferença significativa $p$ valor $<0,05$. 
entre os testes SPV $(8,82 \pm 0,40)$ e CPV $(8,36 \pm 0,50)$. Nos dois exercícios, LP e SR, houve diferença significativa nos resultados.

\section{DISCUSSÃO}

É importante salientar que para conhecermos realmente se uma carga é máxima em um exercício é extremamente necessário que esta carga seja reprodutível através do protocolo de confiabilidade do teste, tornando assim confiáveis também os percentuais estipulados pelo teste de 1-RM. Os coeficientes de relação intra e inter-testes mostraram-se satisfatórios. Isso pode ter ocorrido pelo fato dos exercícios selecionados constarem na rotina de treino dos voluntários.

A utilização da privação visual provocou aumentos significativos nos testes de 1-RM nos exercícios supino reto (SR) $(8,74 \%-p=0,03)$ e leg press $45^{\circ}(\mathrm{LP})(14,04-$ $p=0,001)$. Nos testes com carga de $85 \%$ de $1-R M$ ocorreu também melhora no número de repetições no exercício SR $(22,82 \%-p=0,02)$ e LP $(5,71 \%-p=0,01)$. A PSE também obteve índices significativos de aumento nos exercícios SR $(p=0,03)$ e LP $(p=0,02)$.

Vários estudos utilizam o teste de uma repetição máxima para a avaliação dos níveis de força muscular, o que torna os dados supramencionados ainda mais fidedignos $^{8,19,20}$. Alguns estudos mostram a relação da carga deslocada durante o teste de predição de $1 \mathrm{RM}$ em relação à: familiarização com o teste; com a prática de exercícios resistidos; ângulos articulares envolvidos; tipos de aquecimento (específico ou flexibilidade); e déficit bilateral ${ }^{11,15,19,21}$.

Maior et al ${ }^{3}$., avaliaram e compararam o valor de carga deslocado durante a execução do teste de 1-RM com e sem privação visual nos exercícios Supino Horizontal (SH), Leg Press $45^{\circ}$ (LP) e Puxada pela Frente (PF). Os resultados encontrados na comparação entre os testes com e sem vendas foram: para $\mathrm{SH}, \mathrm{p}=0,0001$; $L P, p=0,0001 ; e P F, p=0,0001$. Resultados semelhantes aos encontrados neste estudo, na comparação entre o supino reto e leg press $45^{\circ}$, foram achados valores relevantes para os exercícios SR $(0,03)$ e LP $(0,001)$. $\mathrm{O}$ aumento de cargas com a privação visual ocorre independente do gênero. Maior et. al ${ }^{14}$, verificaram um aumento na carga relativa e absoluta com a utilização da privação visual no teste de 1-RM nos exercícios Leg Press $45^{\circ}$, Supino Reto e Puxada de Frente em mulheres com experiência no treino de força. De acordo esses dois trabalhos, nossa pesquisa vem corroborar com a literatura no que se refere ao aumento da carga com a utilização da privação visual.

Bandura ${ }^{22}$ acredita que exista uma correlação entre o sistema auto-regulatório (fatores motivacionais e neurais da aprendizagem do movimento) e do processo cognitivo com os estímulos verbais, que auxiliam no controle funcional do indivíduo. Ocorre assim, com o uso de vendas, melhoria dos mecanismos reflexos e princípios homeostáticos, que ajudam no comportamento instintivo que regula as ações motoras através do sistema cognitivo de regulação, o que corrobora com os achados do estudo, pois, durante os testes de 1-RM e de predição de carga ( $85 \%$ de 1-RM) a utilização de vendas nos olhos mostraram aumentos significativos com o uso do estímulo verbal.

Com a privação do sentido da visão, outras fontes sensoriais são utilizadas como o sistema vestibular e somato-sensorial para que diminua as oscilações corporais e diminua a força do acoplamento entre as informações visuais ${ }^{23}$. Não foi observada com o uso da privação em nenhum dos voluntários desvios posturais e de equilíbrio corporal, nem variações no posicionamento das articulações envolvidas nos exercícios utilizados.

Maior et al ${ }^{4}$., acreditam haver uma forte correlação entre a privação visual e o recrutamento de unidades motoras. Assim o individuo aumenta sua força através da coordenação intramuscular, sendo esta responsável pela ativação de todos os músculos envolvidos e maior ativação das unidades motoras.

Porém, nenhum estudo correlacionou o número de repetições e a PSE em relação à predição de carga de 1-RM com ou sem o uso de vendas. Quanto ao número de repetições com $85 \%$ de 1 -RM, observamos um aumento quando realizadas com a privação visual. Segundo Maior et $\mathrm{al}^{4}$, a privação visual pode ter relação com a coordenação intramuscular, com mais unidades motoras sendo recrutadas para a ação muscular. Essa maior coordenação intramuscular pode provocar um aumento na força dos indivíduos, aumentando simultaneamente o número de repetições realizadas nos exercícios LP e SP. Os mecanismos pelos quais a privação visual poderia aumentar o recrutamento das unidades motoras e a coordenação intramuscular ainda não estão esclarecidos. Hodson-Tole et $a^{24,25}$, o recrutamento motor é um fenômeno multifatorial, sendo sua analise uma tarefa complexa e não totalmente conhecido. Outro ponto importante que vale ressaltar é que talvez a utilização do uso de vendas consiga diminuir o efeito do indivíduo subestimar a sobrecarga utilizada durante um dado exercício ${ }^{26}$.

A percepção subjetiva de esforço (PSE) apresentou diferença significativa quando realizada com privação visual comparada a realização sem privação visual. Uma diminuição da PSE pode refletir ao aumento da auto-eficácia cognitiva, proporcionando aos indivíduos uma eficiência na realização das repetições, o que levaria a uma menor percepção para a mesma intensidade. Não foi encontrado na literatura nenhum estudo relacionando PSE e privação visual. 
Este trabalho apresentou algumas limitações tais como: a aplicação dos testes poderia ocorrer em dias alternados para cada exercício; poderiam ter sido investigados mais grupamentos musculares; a falta de um controle sobre o ângulo articular de cada exercício.

\section{CONSIDERAÇÕES FINAIS}

Com os achados deste estudo podemos concluir aumentos significativos no deslocamento de carga (força absoluta e força relativa) nos testes de 1-RM. Na predição de carga ocorreram aumentos relevantes no número de repetições e uma diminuição na PSE na comparação entre os testes com privação visual em relação aos testes sem o uso de vendas. Com isso, verificamos que há mecanismos ainda ocultos que podem aumentar a força muscular, que ainda não foram elucidados pela literatura.

Vale a pena ressaltar que embora os resultados encontrados no presente estudo sejam positivos com o uso da privação visual, mais estudos são necessários para elucidar as variáveis que podem aumentar a força muscular. Sendo assim, novas pesquisas são imprescindíveis, pois a literatura carece de mais informações sobre o assunto abordado.

\section{REFERÊNCIAS}

1. Rhea, M. Alvar, B. Burkett, L. Ball, S. A meta-analysis to determine the dose response for strength development. Medicine Science Sport Exercise. 35(3): 456-464, 2003.

2. ACSM. Position Stand: Progression models in resistance training for healthy adults. Medicine and Science in Sports and Exercise, 41(3), 687-708, 2009.

3. Abadie, B. Wentworth, M. Prediction of one repetition maximal strength from a 5-10 repetition submaximal strength test in college-aged females. JEPonline. 4(2):1-6, 2000.

4. Maior, A. Alves, A. A contribuição dos fatores neurais em fases iniciais do treinamento de força muscular: uma revisão bibliográfica. Revista Motriz. 9(3): 161-168, 2003.

5. Haskell, W. L; Lee, I-M; Pate, R. R; Powell, K. E; Blair, S. N; Franklin, B. A; Macera, C. A; Heath, G. W; Thompson, P. D; Bauman, A. Physical Activity and Public Health: Updated Recommendation for Adults from the American College of Sports Medicine and the American Heart Association. Medicine Science Sports Exercise. 39, 1423-1434, 2007.

6. Pereira A, Izquierdo M, Silva AJ, Costa AM et al. Effects of high-speed power training on functional capacity and muscle performance in older women. Exp Gerontol. 47(3):250-5, 2012.

7. Holviala J, Häkkinen A, Alen M, Sallinen J, Kraemer W, Häkkinen K. Effects of prolonged and maintenance strength training on force production, walking, and balance in aging women and men. Scand J Med Sci Sports, 29, 1333-1340, 2012.

8. Mccurdy, K. Langford, GA. Cline, AL. Doscher, M. Hoff, $\mathrm{R}$. The reliability of 1 and $3 \mathrm{RM}$ test of unilateral strength in trained and untrained men and women. Journal Sport Science Medicine. 3: 190-196, 2004.
9. Kemmler, W. Lauber, D. Engelke, K. Weineck, J. Effect of single Vs. multiple set resistance trainning on maximum strength and body composition in trained postmenopausal women. Journal Streng Cond Resistance. 18 (4): 689-694, 2004.

10. Fleck, SJ. Kraemer, WJ. Fundamentos do Treinamento de Força Muscular, 3 ed. Porto alegre: Artmed, 2007.

11. Chagas, M. Barbosa, JRM. Lima, FV. Comparação do número máximo de repetições realizadas a $40 \%$ e $80 \%$ de uma repetição máxima em dois diferentes exercícios na musculação entre os gêneros masculino e feminino. Revista Brasileira de Educação Física Especializada. 19 (1): 5-12, 2005.

12. Simão, R. Poly, MA. Lemos, A. Prescrição de exercícios através do teste $1 \mathrm{RM}$ em homens treinados. Revista Fitness Performance. 3 (1): 47-52, 2004.

13. Baechle, T. Earle, R. Essentials of strength trainning and condition. Champaign: Human Kinetics, 2000.

14. Maior, AS. Varalo, AT. Matoso, AGPS. Edmundo, DA. Oliveira, MM. Minari, VA. Resposta da força muscular em homens com a utilização de duas metodologias para o teste de 1-RM. Revista Brasileira de Cineantropometria e Desenvolvimento Humano. 2 (2): 117-182, 2007.

15. Moura, J. Borher, T. Prestes, MT. Zinn, JL. Influência de diferentes ângulos articulares obtidos na posição inicial do exercício pressão das pernas e final do exercício puxada frontal sobre os valores de 1-RM. Revista Brasileira de Medicina do Esporte. 10 (4): 269-274, 2004.

16. World Health Organization. Obesity: preventing and managing the global epidemic. Report of the WHO Consultation on Obesity. Geneva: World Health Organization: 1998. 


\section{REFERÊNCIAS}

17. Weinberg, R. Gould, D. Trad. Monteiro, MC. Fundamentos da Psicologia do Esporte e do Exercício. 2. ed. Porto Alegre: Artmed Editora, 2001.

18. Robertson, RJ. Goss, FL. Rutkowski, J. Lenz, B. Dixon, C. Timmer, J. Frazee, K. Dube, J. Andreacci, J. Concurrent Validation of the OMNI Perceived Exertion Scale for Resistence Exercise. Medicine and Science in Sports and Exercise. 35 (2): 333-341, 2003.

19. Dias, R. Cyrino, ES. Salvador, EP. Caldeira, LFS. Nakamura, FY. Papst, RR. Bruna, N. Gurjão, ALD. Influência do processo de familiarização para avaliação da força muscular em testes de 1-RM. Revista Brasileira de Medicina do Esporte. 11 (1): 34-38, 2005.

20. Sakamoto A, Sinclair P. Effect of movement velocity on the relationship between training load and the number of repletion of bench press. Journal Strength Cond Res. 3: 523-527, 2006.

21. Simão, R. Giacomini, MB. Dornelles, TS. Marramom, MGF. Viveiros, LE. Influência do aquecimento específico e flexibilidade no teste de 1-RM. Revista Brasileira de Fisiologia do Exercício. 1(2):134-140, 2003.

22. Bandura, A. Self-Efficacy - The exercise of control. New York: Editora Freeman, 1997

23. Barela, J. Polastri. PF. Freitas Junior, PB. Godoi, D. Efeito da exposição visual no acoplamento entre informação visual e controle postural em bebê. Revista Paulista de Educação Física. 17 (1): 16-31, 2003.

24. Hodson-Tole EF, Walkeling JM. Motor unit recruitment patterns 2: the influence of myoelectric intensity and muscle fascicle strain rate. J Exp Biol. 211 (12): 1893-902, 2008.

25. Hodson-Tole EF, Wakeling JM. Motor unit recruitment patterns 1: responses to change in locomotor velocity and incline. J Exp Biol. 211 (12):1882-92, 2008.

26. George, T. Feltz, D. Motivation in sport from a collective efficacy perspective. Internacional Journal Sport Psyc. 26 (1): 98-116, 1995.

\section{Endereços para correspondência:}

